\title{
Phospho-EGFRTyr992 is synergistically repressed by co-inhibition of histone deacetylase (HDAC) and phosphatidylinositol 3-kinase (PI3K), which attenuates resistance to erlotinib in head and neck cancer cells
}

\author{
Xuejun Chen", Wen Gao", Gaofei Yin, Wei Guo, Junwei Huang, Zhigang Huang, Yang Zhang \\ Department of Otolaryngology Head and Neck Surgery, Beijing Tongren Hospital, Capital Medical University, Beijing, China \\ Contributions: (I) Conception and design: Y Zhang; (II) Administrative support: Z Huang, Y Zhang; (III) Provision of study materials or patients: \\ X Chen, Y Zhang; (IV) Collection and assembly of data: W Gao, G Yin; (V) Data analysis and interpretation: W Guo, J Huang; (VI) Manuscript \\ writing: All authors; (VII) Final approval of manuscript: All authors. \\ \#These authors contributed equally to this work. \\ Correspondence to: Yang Zhang. Department of Otolaryngology Head and Neck Surgery, Beijing Tongren Hospital, Capital Medical University, \\ Beijing 100730, China. Email: zhangyangent@163.com.
}

Background: Erlotinib is a commonly used epidermal growth factor receptor (EGFR)-targeted therapeutic choice for head and neck squamous cell carcinoma; however, its efficacy is largely compromised by cancer cell resistance. Understanding and targeting the erlotinib adaptive mechanisms of squamous cell carcinoma of the head and neck (HNSCC) cancer cells are still pressing challenges. This study aimed to elucidate the cooperative erlotinib-sensitizing mechanisms of histone deacetylase (HDAC) and phosphatidylinositol 3-kinase (PI3K) co-inhibition, which will be helpful in gaining a better understanding of the mechanism of EGFR-tyrosine kinase inhibitor (TKI) resistance in head and neck cancer cells.

Methods: High-content screening (HCS) was performed to analyze the cell counts of different treatment groups and their drug-sensitizing effect phenotype. Western blotting and immunofluorescence staining assays were used to measure and locate the expression of proteins in the FaDu and TU212 cells. Annexin V/ PI and DAPI staining were also used to determine the ratio of apoptotic cells and different cell cycle phases.

Results: The expression of phosphor-EGFRTyr992 was significantly increased in erlotinib-treated FaDu cells compared with dimethyl sulfoxide (DMSO)-treated FaDu cells. Meanwhile, erlotinib + vorinostat + copanlisib jointly attenuated the expression of phosphor-EGFRTyr1068 and phosphor-EGFRTyr992, but stimulated the expression of E-cadherin. Moreover, we found that the tri-drug group also impaired the expression of phosphor-STAT3Ser727 and its relevant activators, including phosphor-SrcTyr416.

Conclusions: These findings indicate that HDACs and PI3K co-inhibition sensitizes erlotinib via inactivation of the phosphor-EGFRTyr1068-induced RTK-STAT3 axis. However, PI3K inhibition was sufficient to sensitize TU212 cells to erlotinib, providing new perspectives for the further clinical study of erlotinib + vorinostat + copanlisib as a potential combination therapeutic solution for EGFR responsive reactivation-induced resistance to erlotinib.

Keywords: Squamous cell carcinoma of the head and neck (HNSCC); erlotinib resistance; combination drug therapeutics

Submitted Jul 23, 2021. Accepted for publication Sep 10, 2021.

doi: $10.21037 /$ atm-21-4335

View this article at: https://dx.doi.org/10.21037/atm-21-4335

(c) Annals of Translational Medicine. All rights reserved. 


\section{Introduction}

Squamous cell carcinoma of the head and neck (HNSCC) is a common malignant tumor in this region, accounting for approximately $7 \%$ of all cancers of the upper aerodigestive tract. In addition, with a high metastatic rate and mortality, HNSCC poses a significant challenge to the development of effective targeted therapeutic solutions that extend the survival time of patients. Epidermal growth factor receptor (EGFR) is aberrantly activated to a considerable extent in HNSCC and imparts a poor prognosis with high rates of recurrence (1).

Currently, erlotinib can halt $\mathrm{FaDu}$ cell proliferation by inhibiting the phosphorylation of EGFR at specific amino acid sites, where these EGFR phosphorylation sites accelerate cell cycling by intracellular signaling to RAS and mitogen-activated protein kinase (MAPK) (2). This initiates the activity of genes such as CDK4 and CCND1, which are involved in the process of cell division (3). However, under erlotinib administration, the median progression-free survival is only 9.6 weeks (95\% CI, 8.1-12.1 weeks), and the median overall survival is only 6.0 months $(95 \%$ CI, $4.8-$ 7.0 months) (4). Despite the wide variety of sophisticated drug adaptation processes that may exert drug resistance, EGFR reactivation under erlotinib intervention is accepted as one of the major mechanisms of drug resistance $(5,6)$. According to the latest researches based on tyrosine phosphoproteomics, actin cytoskeleton and extracellular matrix (ECM)-receptor interaction may also play important roles in the resistance of erlotinib. In addition, histone lysine demethylases are the potential target for erlotinibresistant HNSCC (7). Although combined EGFRLeu858 + EGFRThr790 resistance to erlotinib has been identified as a refractory cancer cell model in non-small cell lung cancer (NSCLC) (5), the novel EGFR reactivation sites have not yet been identified or evaluated.

Phosphatidylinositol 3-kinase (PI3K) is also an aberrantly expressed protein in numerous human tumors, including HNSCC. The high activity of p110 enzymes in PI3K stimulates cell growth, which plays a significant role in pathway compensation when deprived of growth signals from receptor tyrosine kinase (RTK), independent of growth factors (8). PI3K alterations, such as mutation, gene amplification, and overexpression, are common in HNSCC, where gene amplification is the most prevalent type of genetic alteration (approximately $30 \%$ of patients with metastatic HNSCC), and high expression of PI3K indicates poor clinical prognosis (9). As a clinically-approved drug, copanlisib is an efficient panPI3K inhibitor that is highly specific for PI3K isoforms, with more potent suppression of the p110 $\alpha$ and p110 isoforms of PI3K. It decreases the activity of downstream signaling (10), and is often used as an adjuvant of RTK or MAPK inhibitors to generate a cooperative sensitizing effect (11). Currently, copanlisib has exhibited a potential impact in clinical trials with either gain-of-function mutation of PIK3CA or overexpression of human EGFR (6).

Interestingly, histone deacetylases (HDACs) have been found to be overactivated by detecting the hypoacetylation status in HNSCC (12). Knockdown of HDACs could reduce the expression of EGFR by regulating the EGFR activators dissociated from its promoter in colorectal cancer cells (13). Furthermore, it was reported that the chromatin acetylation level was negatively correlated with the clonogenic formation of cancer stem cell (CSC) spheres, which indicated that HDAC inhibition played a role in limiting the CSC population in HNSCC cells (12). In addition, vorinostat can interact and block the catalytic sites of HDACs, slow cell cycling, and promote cellular death of a broad range of cancer cells both in vitro and in tumor-bearing mice at concentrations not harmful to normal cells (14). Clinically, vorinostat has also exhibited a significant tumor suppression capability in doses tolerated by cancer patients with a wide variety of tumors (15).

The aim of this study was to use a combination of adjuvants (vorinostat and copanlisib) to sensitize $\mathrm{FaDu}$ and TU212 cells with erlotinib exposure, and quantify and compare the sensitizing effect among the drug groups.

We present the following article in accordance with the MDAR reporting checklist (available at https://dx.doi. org/10.21037/atm-21-4335).

\section{Methods}

\section{Cell culture and administration of drug combinations}

The human hypopharyngeal cancer cell line, $\mathrm{FaDu}$, was obtained from the American Type Culture Collection (ATCC), American. The human laryngeal cancer cell line, TU212, was acquired from Beijing Zhongke Quality Inspection Biotechnology Co. Ltd. (Beijing, China). The cells were cultured in RPMI 1640 medium with $10 \%$ fetal bovine serum (FBS). Erlotinib (E), vorinostat (V), and copanlisib (C) were provided by Selleck Inc. (Shanghai, China), and diluted in 10\% FBS RPMI 1640 medium at working concentrations (erlotinib: $10 \mu \mathrm{M}$; vorinostat: $1 \mu \mathrm{M}$; copanlisib: 90, $120 \mathrm{nM}$ ) for a $72 \mathrm{~h}$ combination drug intervention. The treatment groups were designated as follows: (I) erlotinib + vorinostat + copanlisib; (II) erlotinib 
+ vorinostat; (III) erlotinib + copanlisib; (IV) erlotinib; (V) vorinostat + copanlisib; (VI) vorinostat; (VII) copanlisib; and (VIII) dimethyl sulfoxide (DMSO).

\section{High-content screening (HCS) assay and quantification of the erlotinib sensitizing effect}

For combination drug screening, FaDu cells were plated out to a final density of 15,000 cells/well (for $24 \mathrm{~h}$ treatment) in CellCarrier 96-well plates (PerkinElmer, American). The cells were incubated overnight at $37{ }^{\circ} \mathrm{C}$ in a humidified atmosphere with $5 \% \mathrm{CO}_{2}$ and $95 \%$ air. On the following day, the small molecule inhibitors to be tested were then added according to the treatment groups (erlotinib + vorinostat + copanlisib, erlotinib + vorinostat, erlotinib + copanlisib, erlotinib, vorinostat + copanlisib, vorinostat, copanlisib, and DMSO) to a final dose range of $0.01-100 \mu \mathrm{M}$ at a final concentration in supplemented RPMI 1640 medium in each well. All drugs were acquired from Selleck Inc. and were of the highest quality possible. Small molecule compound precipitation was checked by visual examination of stock solutions after the dilution series had been transferred into supplemented RPMI 1640 medium, and then incubated with cells for $72 \mathrm{~h}$. No visible precipitation was observed for any of the testing chemicals.

After $72 \mathrm{~h}$ of protein intervention by different small molecule inhibitor groups, the culture medium was aspirated and carefully replaced with $100 \mu \mathrm{L}(1 \mu \mathrm{g} / \mathrm{mL})$ Hoechst33342 and propidium iodide (PI) (Solarbio, Beijing, China). The plates were incubated for $30 \mathrm{~min}$ at $25^{\circ} \mathrm{C}$ and then gently washed three times with pre-warmed PBS, leaving $100 \mu \mathrm{L}$ after the last washing step. For fixation, $50 \mu \mathrm{L} /$ well of $4 \%$ paraformaldehyde (Beyotime Biotechnology, Nantong, China) was added, followed by a $30 \mathrm{~min}$ incubation at $25^{\circ} \mathrm{C}$. The cells were then washed three times with PBS in preparation for HCS imaging. Twenty-one image fields per well were acquired using the PerkinElmer Operetta CLS Reader with an LED light engine, using a NA 0.75 10x air objective. The nuclei fluorescence of Hoechst 33342 was used for automatic focusing.

Image analysis was performed using the Harmony 4.5 (PerkinElmer). The Hoechst33342 parameters of nuclei counts and nuclear area were acquired from the $490 / 360 \mathrm{~nm}$ emission/excitation channel, and the PI parameter of death counts was acquired from the $650 / 590 \mathrm{~nm}$ emission/ excitation channel. The live cell population was selected from the whole population, including apoptotic and dead cells. From the HCS-generated live cell count data $(\mathrm{N})$, the phenotypic $\lambda$ was used as the metric to quantify the erlotinib sensitizing effect conferred by drug $\mathrm{X}$, which was calculated as follows:

$$
\lambda(\mathrm{X})=\frac{\frac{N(X) \text { Erlotinib }}{N(X) D M S O}}{\frac{N \text { Erlotinib }}{N D M S O}}
$$

$\lambda(X)>0$ denotes the effect of resistance and $\lambda(X)<0$ denotes the effect of sensitizing.

The cooperative effect was determined from the statistically significant difference in $\lambda$ between the different treatment groups, which was evaluated using the two-tailed Student's $t$-test.

\section{Western blot assay}

Cell lysates were extracted with RIPA lysis buffer (Beyotime), and the abundance of protein in samples was determined using the BCA Protein Assay Kit (Beyotime). A $20 \mu \mathrm{g}$ aliquot of each protein sample was separated by sodium dodecyl sulfate polyacrylamide gel electrophoresis and transferred to $0.45 \mu \mathrm{m}$ PVDF membranes (Beyotime). After blocking for $1 \mathrm{~h} 30 \mathrm{~min}$, the membranes were treated overnight at $4{ }^{\circ} \mathrm{C}$ with the primary antibodies of phosphor-EGFRTyr1068, phosphor-EGFRTyr992, phosphor-SrcTyr416, phosphor-AK2Tyr1007, and Survivin. Membranes were washed with Tris-buffered saline with Tween (TBST; Solarbio) and incubated for $1 \mathrm{~h}$ at $25^{\circ} \mathrm{C}$ with horseradish peroxidase (HRP)-conjugated secondary antibodies (Beyotime). Protein bands were detected using a chemiluminescence detection kit (Beyotime). The optical densities of protein signals were calculated using ImageJ software (National Institutes of Health, American) and $\beta$-actin was used as the loading control.

\section{Immunofluorescence staining}

The cells with the various combination drug treatments were grown for $72 \mathrm{~h}$ on glass coverslips in the wells of 24-well plates. They were then fixed with $4 \%$ paraformaldehyde for $10 \mathrm{~min}$ at room temperature. Subsequently, the antigen retrieval buffer (Beyotime) was preheated to $95^{\circ} \mathrm{C}$ and the buffer was heated in a coverglass staining jar, which was placed in a water bath at $95^{\circ} \mathrm{C}$. The coverslips were removed from the antigen retrieval buffer and then immersed in TBST with the side containing the cells facing upwards. The cells were then washed three times for $5 \mathrm{~min}$ in TBST. The samples were subsequently 
incubated for 10 min with TBST containing either $0.25 \%$ Triton X-100 (Beyotime) or 0.5\% saponin (Beyotime) for protein expression. Next, the cells were washed three times for $5 \mathrm{~min}$ in TBST, and were then incubated with $1 \%$ BSA for $1 \mathrm{~h}$ to block any binding of antibodies to impurities.

The cells in the paired $100 \times$ diluted antibodies were incubated overnight at $4{ }^{\circ} \mathrm{C}$ in $1 \% \mathrm{BSA}$ in a humidified chamber. Next, the cells were washed three times for $5 \mathrm{~min}$ in TBST and incubated with both secondary antibodies in $1 \% \mathrm{BSA}$ for $1 \mathrm{~h} 30 \mathrm{~min}$ at $25^{\circ} \mathrm{C}$ in the dark. The cells were then washed three times for $5 \mathrm{~min}$ in TBST followed by DAPI staining for $5 \mathrm{~min}$ at $25^{\circ} \mathrm{C}$ in the dark. The cells were again washed three times for 5 minutes in TBST. The coverslips were mounted with a drop of mounting medium, and imaged using a Zeiss confocal microscope (Germany) over the coverslips in separate channels with $\mathrm{Cy} 3$, fluorescein isothiocyanate (FITC), and DAPI.

\section{Analysis of apoptosis}

Briefly, cells were incubated at $37{ }^{\circ} \mathrm{C}$ for $48 \mathrm{~h}$ in culture medium alone or containing drug regimens. They were then harvested in cold PBS and washed with PBS once, and subsequently incubated with binding buffer at room temperature for $10 \mathrm{~min}$ in PBS containing Annexin V. Next, the cells were resuspended in $1 \mathrm{~mL}$ of PI staining reagent (50 $\mu \mathrm{L}$ propidium iodide in $1 \mathrm{~mL}$ of sodium citrate buffer, $\mathrm{pH}$ 7.4). The samples were then incubated in the dark for another $10 \mathrm{~min}$ before cell cycle analysis. The distribution of cells in the four quadrants was measured and analyzed using the Beckon Dickinson FACS analysis system and FlowJo software (American).

\section{Cell cycling analysis}

The cells were incubated at $37^{\circ} \mathrm{C}$ for $24 \mathrm{~h}$ in culture medium alone or containing drug regimens. They were then harvested in cold PBS, fixed in $70 \%$ ethanol, and stored at $4{ }^{\circ} \mathrm{C}$ for subsequent cell cycle analysis. Fixed cells were washed once with PBS and incubated cells with binding buffer were washed at room temperature for $10 \mathrm{~min}$ in PBS containing Annexin V. Next, the cells were resuspended in $1 \mathrm{~mL}$ of PI staining reagent $(50 \mu \mathrm{L}$ propidium iodide in $1 \mathrm{~mL}$ of sodium citrate buffer, $\mathrm{pH}$ 7.4). Samples were incubated in the dark for another $10 \mathrm{~min}$ before cell cycle analysis. The distribution of cells in the cell cycle was measured using the Beckon Dickinson FACS analysis system and quantified using ModFit software (Verity
Software House, American). The percentages of cells in the G1, S, and G2/M phases were calculated.

\section{Statistical analysis}

Data were reported as means \pm SD from triplicate experiments. The two-tailed Student's $t$-test was used to determine the statistical significance between two groups with $\mathrm{P}$ values $<0.05$ considered to be statistically significant. Analysis was performed using GraphPad Prism 7 (GraphPad Prism Software Inc., San Diego, CA, USA).

\section{Results}

Combined use of adjuvant copanlisib + vorinostat synergistically sensitized the erlotinib resistance of FaDu and TU212 cells

Using a HCS system, the count of FaDu cells in each treatment group was scanned and recorded (Figure 1A). According to the formula $\lambda(\mathrm{X})$ described in the Methods section, we found that vorinostat or copanlisib had a sensitizing effect on the $\mathrm{FaDu}$ cells under erlotinib pressure. Moreover, the combined use of the vorinostat $(1 \mu \mathrm{M}$ as working concentration) + copanlisib (90 or $120 \mathrm{nM}$ as working concentration) exerted a synergistic sensitizing effect on erlotinib that was statistically significant compared to the adjuvant use of vorinostat or copanlisib alone (Figure 1B). We further validated the synergistic sensitizing effect on the TU212 cell line by detecting its cell vitality with exposure to these combination drug regimens (Figure 1C).

\section{Erlotinib + copanlisib + vorinostat downregulated erlotinib-irresponsive phosphor-EGFRTyr992 in the FaDu and TU212 cell lines}

To explore the mechanism of resistance to erlotinib, we first tested the expression of phosphor-EGFRTyr 1068 and phosphor-EGFRTyr992 in the erlotinib-treated and DMSO-treated groups, as shown in Figure $2 A, 2 B$, respectively. Phosphor-EGFRTyr1068 decreased significantly under erlotinib administration compared with the DMSO group $(\mathrm{P}<0.01$, Figure $2 A)$. However, we found that phosphor-EGFRTyr992 was significantly increased in the erlotinib group compared with the DMSO group $(\mathrm{P}<0.05$, Figure $2 B)$, indicating that erlotinib may not be an effective inhibitor at the phosphorylation site of EGFR Tyr992 and that phosphor-EGFRTyr992 might play a compensatory role in EGFR reactivation. 
A

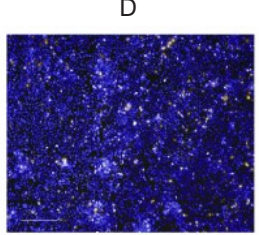

E

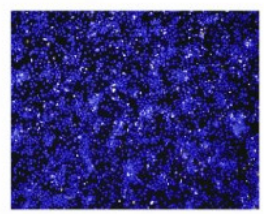

$D+V$

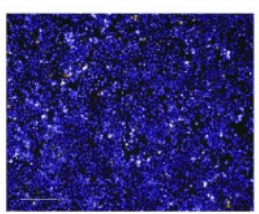

$E+V$

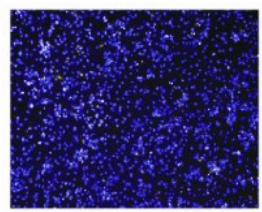

$D+C$

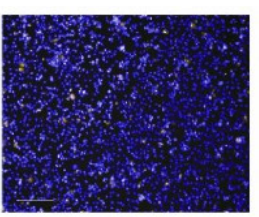

$E+C$
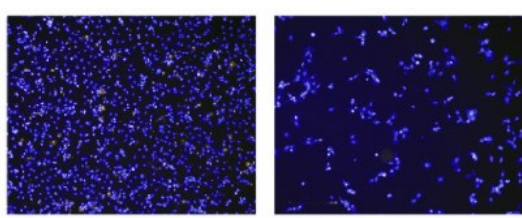

B
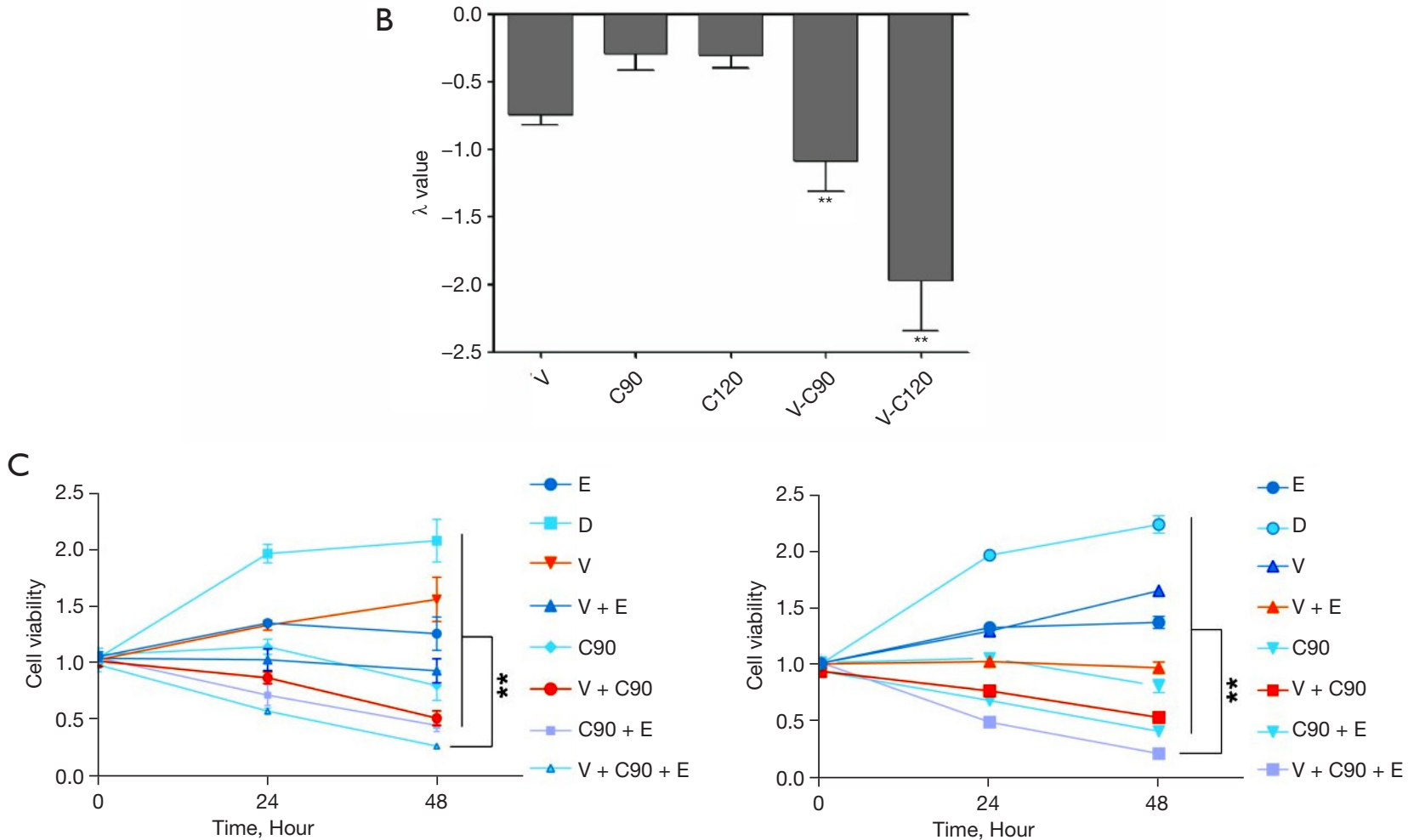

Figure 1 Copanlisib + vorinostat synergistically enhanced the sensitization of the FaDu cells under erlotinib pressure (DMSO, erlotinib, vorinostat, and copanlisib are abbreviated as D, E, V, and C, respectively). (A) HCS morphological demonstration of FaDu cells in the combination drug treatment groups, Annexin V/PI staining, 10×4 times. (B) The $\lambda$ value of each drug group was quantified and compared using the two-tailed Student's $t$-test. Data are presented as means $\pm \mathrm{SD},{ }^{* *} \mathrm{P}<0.01$ compared with the erlotinib group. (C) Vitality analyses of the TU212 cell line were performed under combination drug interventions of D, E, V, and C. Drug effects were compared using the paired $t$-test. Data are presented as means $\pm \mathrm{SD},{ }^{* *} \mathrm{P}<0.01$ compared with the erlotinib + copanlisib + vorinostat combination. DMSO, dimethyl sulfoxide; HCS, high-content screening.

Furthermore, we detected the protein expression of phosphor-EGFRTyr 1068 and phosphor-EGFRTyr992 in other combination treatment groups. Surprisingly, we observed that phosphor-EGFRTyr1068 and phosphorEGFRTyr992 were both downregulated in the tri-drug group compared with the erlotinib group (Figure 2A,2B), suggesting that the erlotinib sensitizing effect was mainly due to suppression of the phosphorylation level of EGFR, especially the erlotinib-insensitive EGFR phosphorylation sites (such as Tyr992 of EGFR) (Figure 2B). In addition, we 


\section{A} p-EGFRTyr1055 Actin

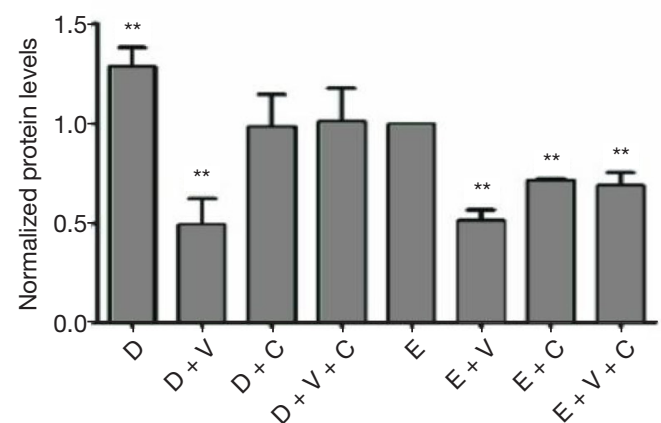

C

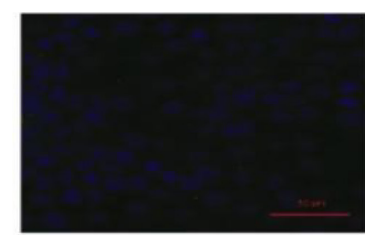

E

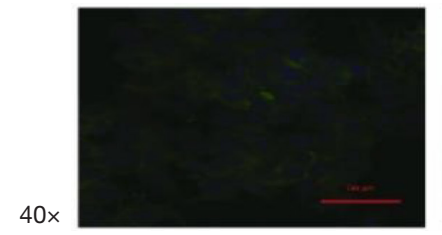

D

D

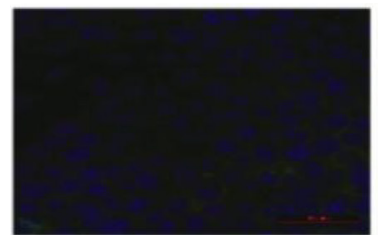

E

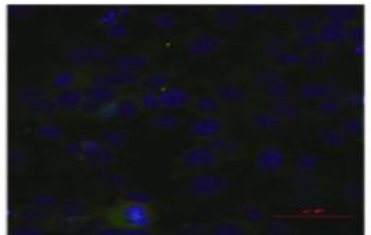

$E+V$
$B$

p-EGFRTyr99
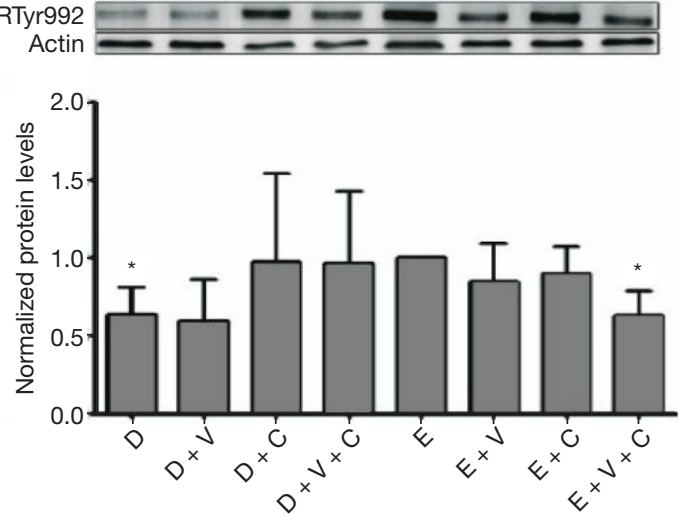

$D+C$
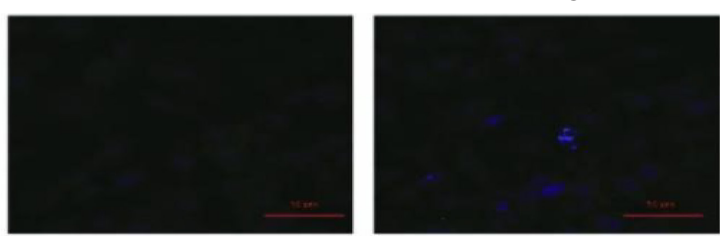

$E+C$

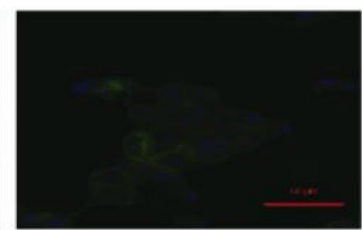

$D+C$

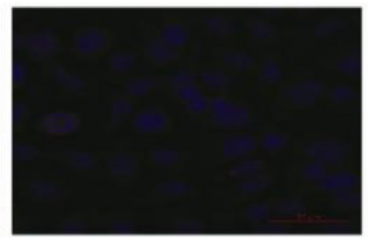

$E+C$

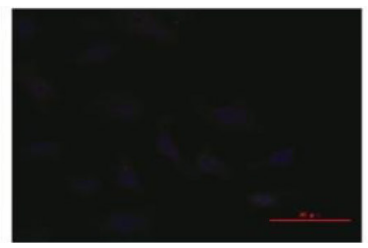

$D+V+C$

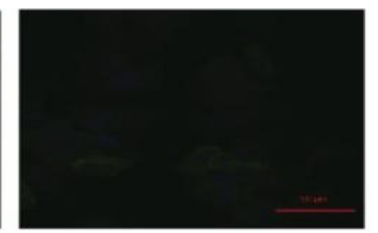

$E+V+C$

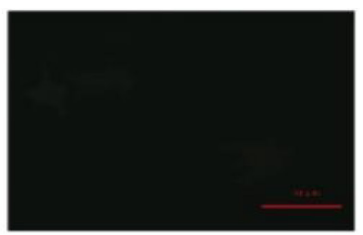

$D+V+C$

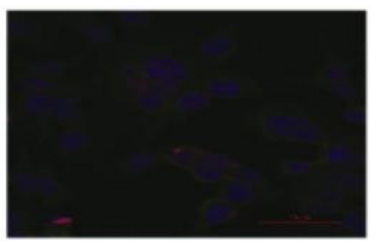

$E+V+C$

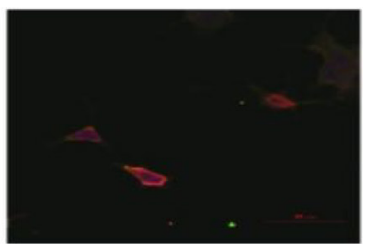




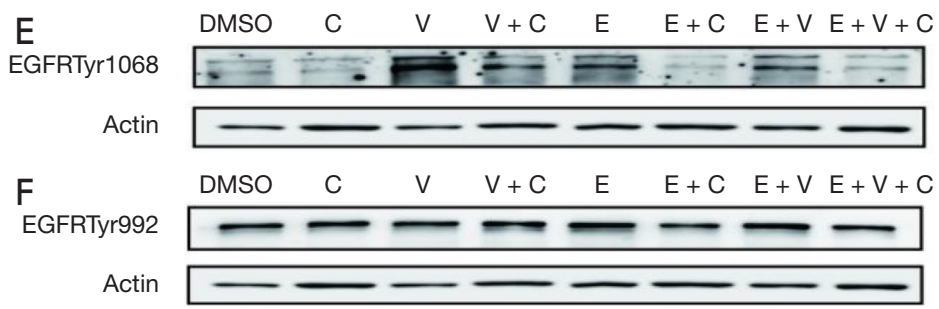

Figure 2 Phosphor-EGFRTyr1068, phosphor-EGFRTyr992, and E-cadherin expression levels in the different treatment groups (DMSO, erlotinib, vorinostat, and copanlisib are abbreviated as D, E, V, and C, respectively). (A) The phosphor-EGFRTyr1068 expression in drugtreated FaDu cells was detected by western blot analysis. $\beta$-actin was also detected as the control for sample loading. Data are presented as means $\pm \mathrm{SD},{ }^{* *} \mathrm{P}<0.01$ compared with the erlotinib group. (B) The phosphor-EGFRTyr992 expression in drug-treated FaDu cells was detected by western blot analysis. $\beta$-actin was also detected as the control for sample loading. Data are presented as means $\pm \mathrm{SD},{ }^{*} \mathrm{P}<0.05$, ${ }^{* *} \mathrm{P}<0.01$ compared with the erlotinib group. (C) The phosphor-EGFRTyr992 (FITC-Green) expression in drug-treated FaDu cells was detected by immunofluorescence analysis, DAPI staining, 10×40 times. (D) The E-cadherin (Cy3-Red) + KLF4 (FITC-Green) expression in drug-treated FaDu cells was detected by immunofluorescence analysis, DAPI staining, 10×40 times. (E) The phosphor-EGFRTyr1068 expression in drug-treated TU212 cells was detected by western blot analysis. $\beta$-actin was also detected as the control for sample loading. (F) The phosphor-EGFRTyr992 expression in drug-treated TU212 cells was detected by western blot analysis. $\beta$-actin was also detected as the control for sample loading. EGFR, epidermal growth factor receptor; DMSO, dimethyl sulfoxide.

also morphologically detected the phosphor-EGFRTyr992, and E-cadherin-KLF4 protein expression within the combination treatment samples. Interestingly, phosphorEGFRTyr992 was abrogated in the tri-drug group, while its expression within the cell membrane of erlotinib was obvious (Figure 2C). E-cadherin was also significantly restored at the cell membrane in the tri-drug treated $\mathrm{FaDu}$ cells, and KLF4 was removed from the cytoplasm (Figure 2D) to the other groups. These results indicated that reversal of the malignant transformation event could be triggered by the synergistic effect of using vorinostat and copanlisib with erlotinib administration.

Although the expression of phosphor-EGFRTyr992 was not observed in the TU212 cell line (Figure 2E), phosphorEGFRTyr1068 was significantly downregulated (Figure $2 F$ ). Taken together, the tri-drug group not only exhibited an effective suppression of the EGFR phosphorylation level, but also promoted an epithelial-mesenchymal transition (EMT)-reversing effect.

\section{Erlotinib-induced inflammatory pro-survival signals of phosphor-7AK2Tyr1007 and phosphor-STAT3Ser 727 were compromised by additive use of the adjuvant combination of copanlisib + vorinostat}

Subsequently, we investigated the tri-drug effect on the pro-survival pathway of the Janus-activated kinase (JAK)-STAT3 axis in both FaDu and TU212 cell lines.
As shown in Figure $3 A$ and $3 \mathrm{~B}$, although erlotinib could moderately decrease the expression of phosphorJAK2Tyr1007 and phosphor-STAT3Ser727, the tri-drug group had a stronger suppression effect, which significantly abrogated the expression of phosphor-JAK2Tyr1007 and phosphor-STAT3Ser727 (Figure 3A,3B). Notably, the use of vorinostat alone also had an abrogative effect on the expression of phosphor-JAK2Tyr1007; however, the effect was buffered when paired with copanlisib or erlotinib alone (Figure $3 A, 3 B$ ). We also found that copanlisib epistatically lowered the level of phosphor-STAT3Ser727 in the tri-drug group (Figure 3C,3D). Thus, these results indicated that the tri-drug group had a combination advantage in reducing the pro-survival signal of phosphor-STAT3Ser727.

\section{Downregulation of phosphor-SrcTyr41 and Survivin by the combined use of erlotinib + copanlisib + vorinostat}

We also detected the expression of phosphor-Src and Survivin, which was reported to be positively associated with the expression of phosphor-STAT3, in order to study the potential downstream effector that correlated with the synergistic cooperation $(16,17)$. We discovered that phosphor-SrcTyr416 and Survivin had been significantly compromised in the tri-drug group compared with the erlotinib group $(\mathrm{P}<0.01$, Figure 4$)$. It is worth noting that copanlisib had an epistatic effect on the influence of phosphor-SrcTyr416 in the erlotinib + copanlisib group, 


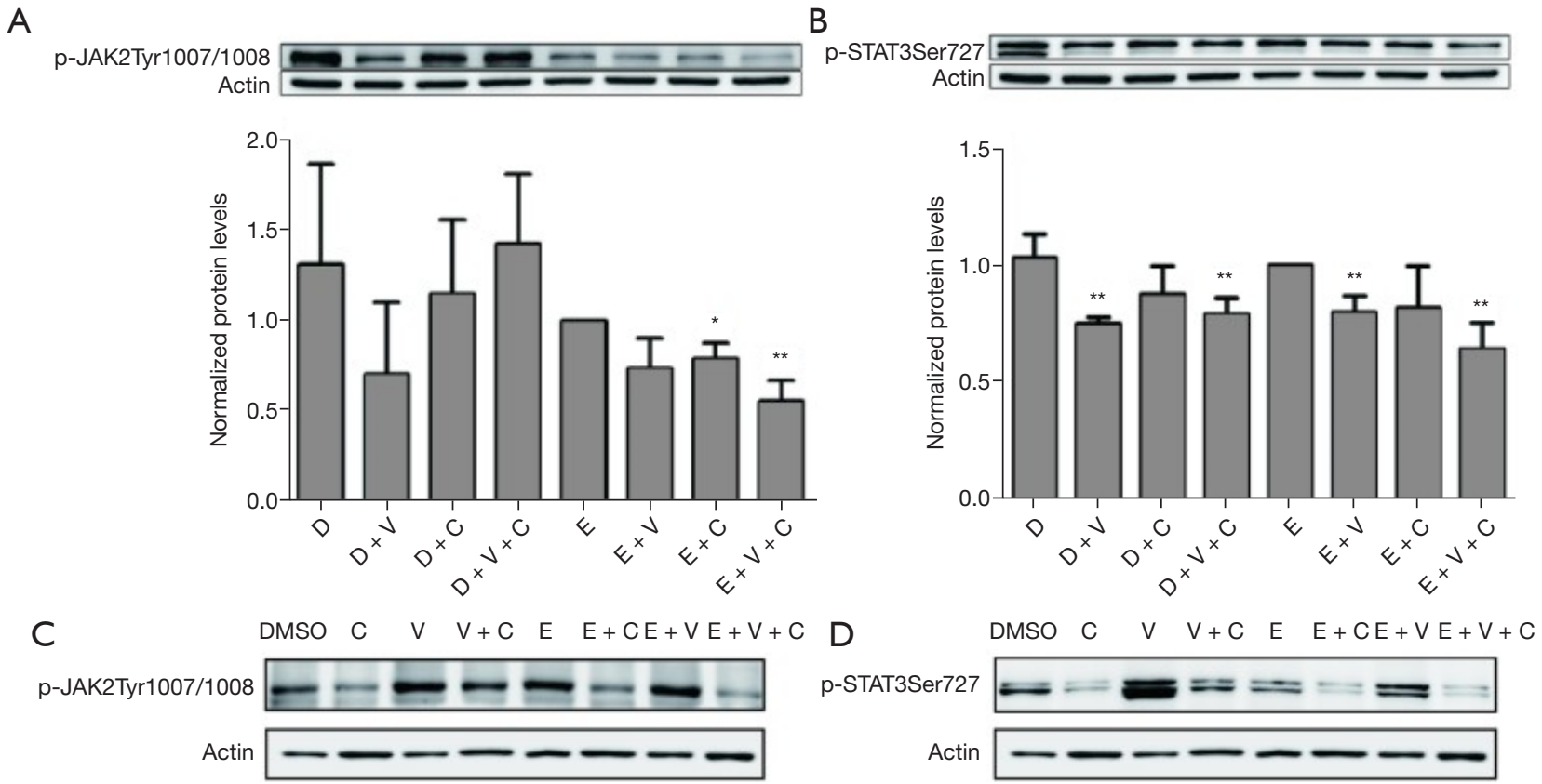

Figure 3 Phosphor-JAK2Tyr1007 and phosphor-STAT3Ser727 expression levels in different treatment groups (DMSO, erlotinib, vorinostat, and copanlisib are abbreviated as D, E, V, and C, respectively). (A) The phosphor-JAK2Tyr1007 expression in drug-treated FaDu cells was detected by western blot analysis. $\beta$-actin was also detected as the control for sample loading. Data are presented as means $\pm \mathrm{SD}$ ${ }^{*} \mathrm{P}<0.05,{ }^{* *} \mathrm{P}<0.01$ compared with the erlotinib group. (B) The phosphor-STAT3Ser727 expression in drug-treated FaDu cells was detected by western blot analysis. $\beta$-actin was also detected as the control for sample loading. Data are presented as means $\pm \mathrm{SD},{ }^{* *} \mathrm{P}<0.01 \mathrm{compared}$ with the erlotinib group. (C) The phosphor-JAK2Tyr1007 expression in drug-treated TU212 cells was detected by western blot analysis. $\beta$-actin was also detected as the control for sample loading. (D) The phosphor-STAT3Ser727 expression in drug-treated TU212 cells was detected by western blot analysis. $\beta$-actin was also detected as the control for sample loading. DMSO, dimethyl sulfoxide.

and maintained the expression of phosphor-SrcTyr416 at a low level. With the addition of copanlisib, the buffering effect of Survivin induced by the interference of erlotinib in vorinostat had been abrogated. In contrast, in TU212 cells, copanlisib epistatically suppressed the expression of phosphor-SrcTyr416 and Survivin. Taken together, although erlotinib + copanlisib was sufficiently efficacious for the protein inhibition of phosphor-SrcTyr416 and Survivin in the TU212 cell line, in FaDu cells, the expressions of phosphor-SrcTyr416 and Survivin were only significantly blocked in the tri-drug group, in addition to the inhibition of the effect of phosphor-JAK2Tyr1007, to cooperatively limit the activity of STAT3.

\section{The synergistic effect of erlotinib + copanlisib + vorinostat was associated with the joint effects of cell cycle inbibition and promotion of cellular apoptosis}

Crucially, the combined use of erlotinib + copanlisib + vorinostat was superior to using any combination of two of these drugs. In this study, we found that, although the apoptotic rates of $\mathrm{E}+\mathrm{C}$ or $\mathrm{E}+\mathrm{V}$ showed no apparent difference from the erlotinib + copanlisib + vorinostat group (Figure $5 \mathrm{~A}$ ), the cell cycling rate was accelerated in $\mathrm{E}+\mathrm{C}$ and $\mathrm{E}+\mathrm{V}$ groups, which neutralized the combination effect of apoptosis. However, vorinostat combined with copanlisib in the erlotinib environment buffered the accelerated cell cycling rate brought about by the combined use of $\mathrm{E}+\mathrm{C}$ and $\mathrm{E}+$ $\mathrm{V}$ on the enrichment of the $\mathrm{S}$ and G2/M phases (Figure $5 B$ ), while achieving an excellent apoptotic rate. At the cellular phenotype level, our cell cycling analysis combined with the apoptotic effects illustrated the mechanism through which the synergistic sensitizing action was triggered by using the tridrug combination of erlotinib + copanlisib + vorinostat.

\section{Discussion}

At present, erlotinib is a commonly used clinical agent for 

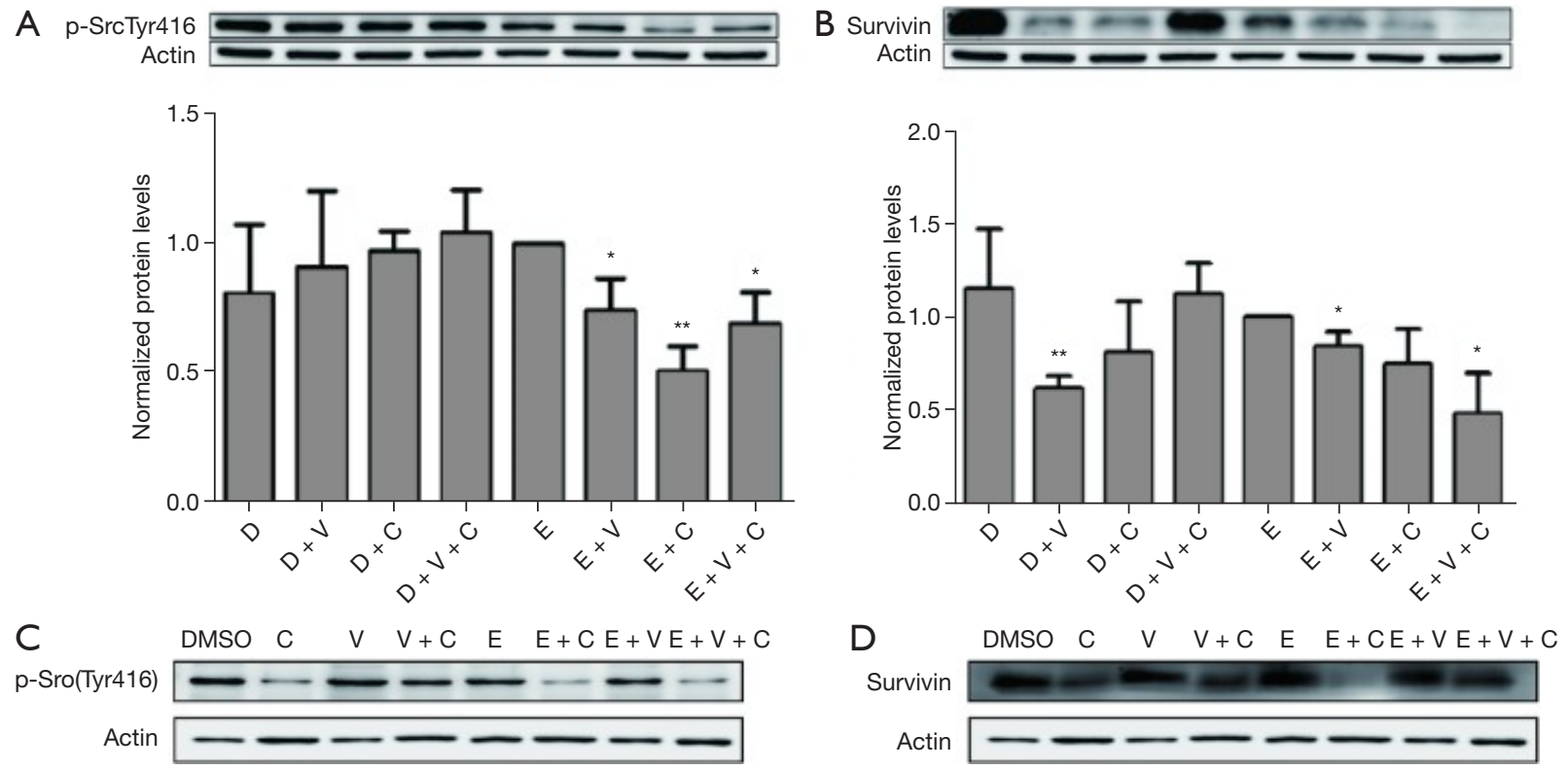

Figure 4 Phosphor-SrcTyr416 and Survivin expression levels in the different treatment groups (DMSO, erlotinib, vorinostat, and copanlisib are abbreviated as D, E, V, and C, respectively). (A) The phosphor-SrcTyr416 expression in drug-treated FaDu cells was detected by western blot analysis. $\beta$-actin was also detected as the control for sample loading. Data are presented as means $\pm \mathrm{SD},{ }^{*} \mathrm{P}<0.05$, ${ }^{* *} \mathrm{P}<0.01$ compared with the erlotinib group. (B) The Survivin expression in drug-treated FaDu cells was detected by western blot analysis. $\beta$-actin was also detected as the control for sample loading. Data are presented as means $\pm \mathrm{SD},{ }^{*} \mathrm{P}<0.05,{ }^{* *} \mathrm{P}<0.01$ compared with the erlotinib group. (C) The phosphorSrcTyr416 expression in drug-treated TU212 cells was detected by western blot analysis. $\beta$-actin was also detected as the control for sample loading. (D) The Survivin expression in drug-treated TU212 cells was detected by western blot analysis. $\beta$-actin was also detected as the control for sample loading. DMSO, dimethyl sulfoxide.

EGFR inactivation in HNSCC (4). It plays a major role in suppressing the phosphorylation site of EGFR Leu858 and the auto-phosphorylation site of Tyr1068, where upregulation of phosphor-EGFRTyr1068 expression is most frequently detected in HNSCC and NSCLC patients (18). On the other hand, EGFRTyr1068 is a distinctly recognized biomarker of lung CSCs (19). However, resistance to erlotinib has been widely reported in HNSCC and NSCLC patients $(20,21)$.

Various mechanisms have been identified as responsible for cancer cell survival under EGFR inactivation. As a major causative mechanism of resistance to erlotinib, the emergence of a "gatekeeper" mutation or the presence of a secondary unaffected phosphorylation site would confer increased downstream transduction strength in EGFR (22). For example, the EGFRThr790Met mutant could exert a strengthening effect on the affinity of adenosine triphosphate (ATP) to maintain the phosphorylation level (5).

In addition, by taking advantage of EGFR addiction, HDAC inhibitor was found to be a positive regulator of E-cadherin and could cause increased expression of phosphorylated EGFR in lung cancer cells, eventually producing an increased EGFR tyrosine kinase inhibitor (TKI) response (23). Meanwhile, knocking down of E-cadherin has been shown to decrease the level of EGFR phosphorylation, and induce resistance to erlotinib treatment (23). Interestingly, our results showed similar effects when we tested the expression of E-cadherin under the adjuvant co-inhibition of HDAC and PI3K with exposure to erlotinib.

Moreover, the PI3K pathway has a significant impact on the mechanistic target of rapamycin (mTOR) to facilitate the pro-survival signals of cancer cells under the inactivation of RTK (24), and could also act as a bypass route to positively promote cell cycling independent of extracellular growth factors. Notably, although the sole inhibition of PI3K activity has a modest antitumor effect, this nevertheless could be effective in a wide range of antitumor therapeutic applications when paired with another antitumor drug (11). In this study, the positive $\mathrm{FaDu}$ sensitizing effect of $\mathrm{PI} 3 \mathrm{~K}$ inhibition 


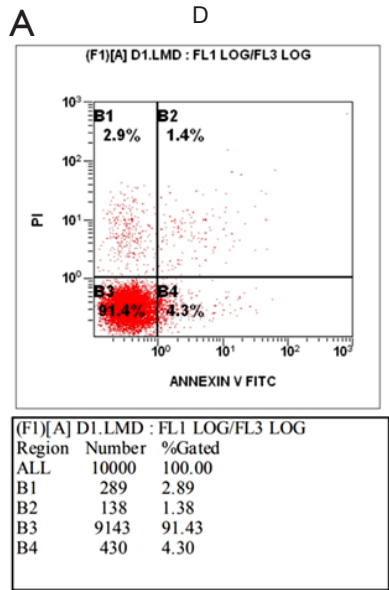

E

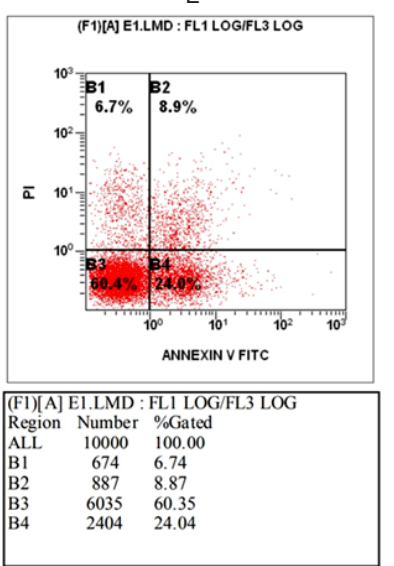

B
V

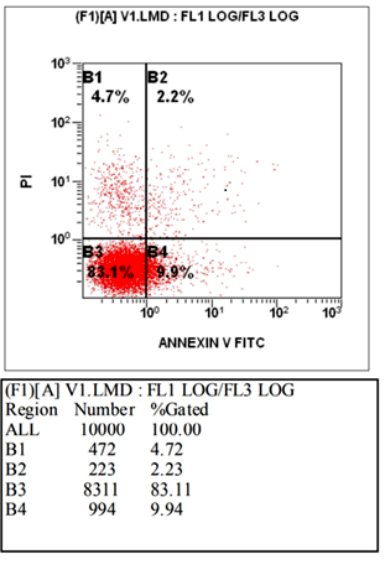

$E+V$
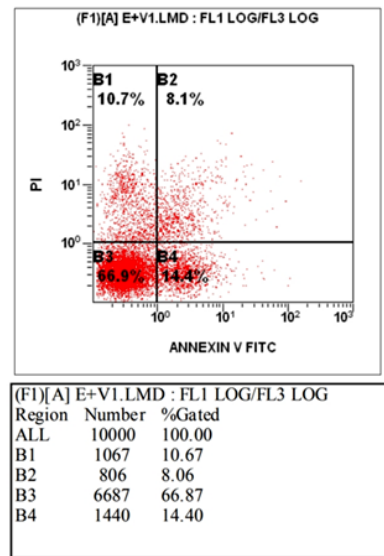

v

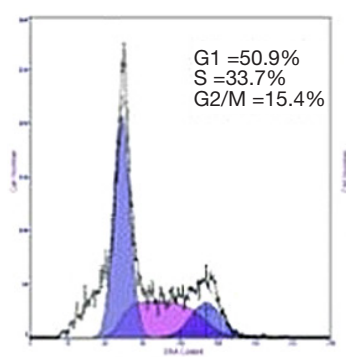

$\mathrm{E}$
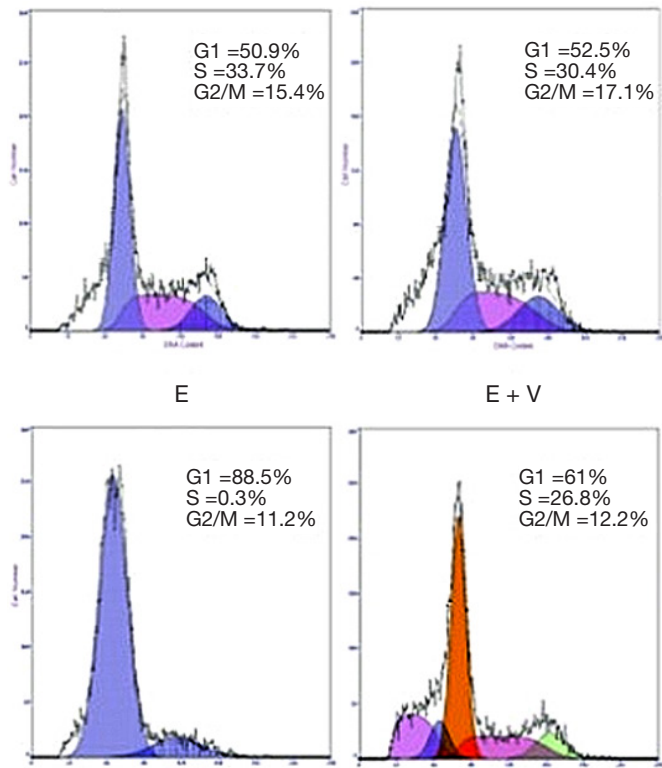

$E+V$

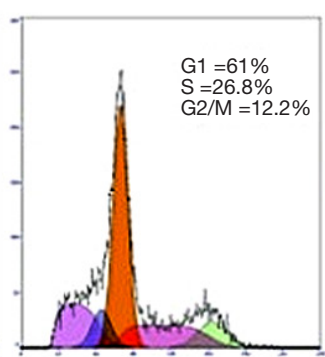

C

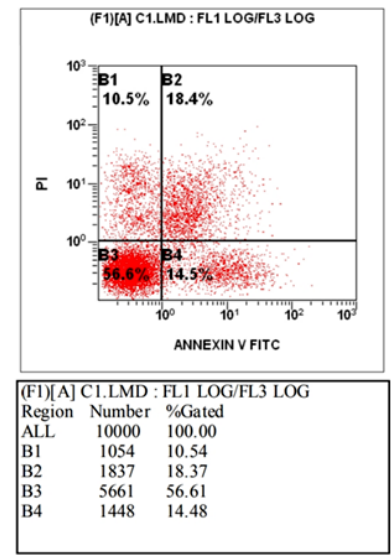

$E+C$
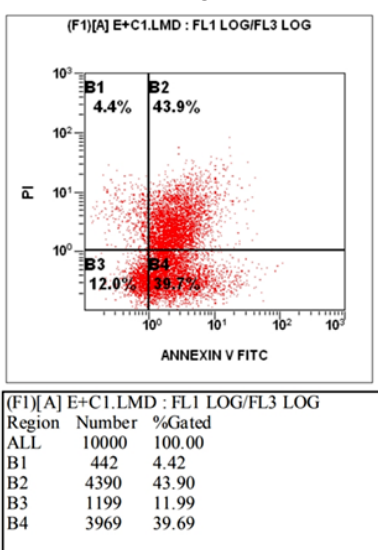

C

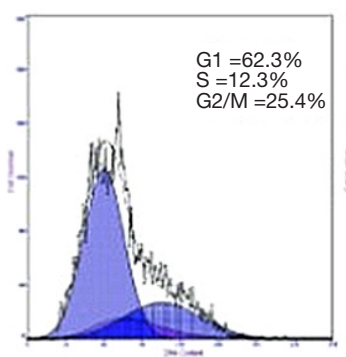

$E+C$

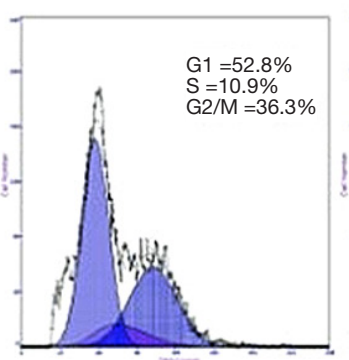

$\mathrm{V}+\mathrm{C}$

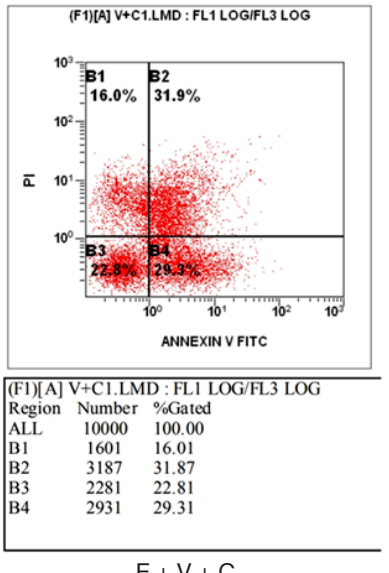

$E+V+C$

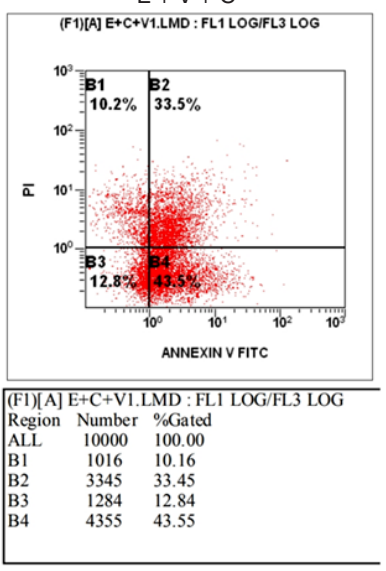

$V+C$

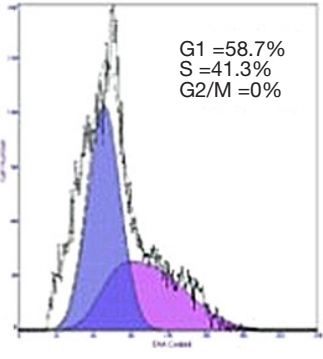

$E+V+C$

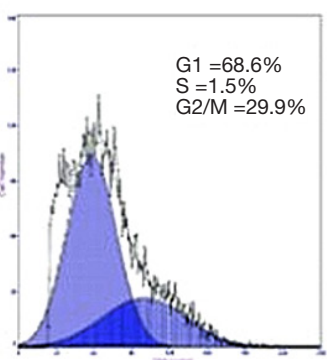

Figure 5 The tri-drug synergistic effect of erlotinib sensitization was induced by enhancing apoptosis and masking the side effect of accelerated cell cycling at the cellular level. (A) Apoptotic analysis of FaDu cells under combination treatments using Annexin V and PI. (B) Cell cycle analysis of $\mathrm{FaDu}$ cells under combination treatments using FACS. 


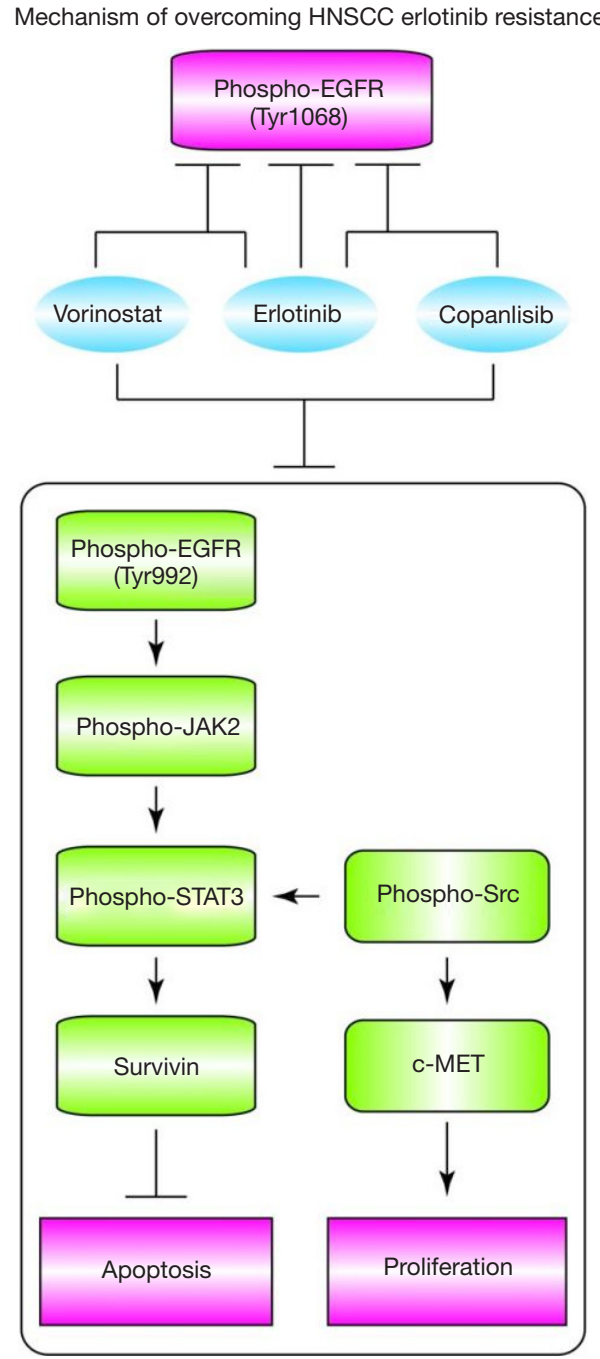

Figure 6 Mechanism for overcoming HNSCC erlotinib resistance. HNSCC, squamous cell carcinoma of the head and neck.

under EGFR-TKI administration was confirmed, and the combined mechanism with erlotinib and HDAC revealed its role in modulating the erlotinib refractory phosphorylation site of EGFR.

As a member of the STAT family of transcription factors, STAT3 is widely activated in several cancers. STAT3 tyrosine phosphorylation can be induced by the stimulation of upstream kinases, such as JAK, EGFR, Survivin, and Src family kinases $(16,17,25)$. Phosphor-STAT3Ser727 has been reported to have a variable response to erlotinib administration (26). Therefore, elevation of phosphorSTAT3 has been identified in the resistance mechanism of EGFR TKIs in HNSCC. Our experiment identified that the tri-drug group of erlotinib, vorinostat, and copanlisib had a significant suppression effect on phosphor-STAT3. These results indicated that the sensitizing effect at the molecular level was mainly based on the combined decrease of phosphor-EGFRTyr992 and phosphor-STAT3, which disrupted a set of passenger signals promoting cell survival. On the other hand, phosphor-Src was reported to be an activator of c-MET, which further initiates accelerated proliferation (21).

In contrast to TU212, FaDu cells were refractory to the use of erlotinib + copanlisib without the addition of vorinostat. We attribute this phenomenon to a change in activation of another phosphor-EGFR site, such as phosphor-EGFRTyr992. To suppress this adaptive drug response, vorinostat exhibited an essential buffering function with copanlisib when using erlotinib in the prevention of cell cycle progression in $\mathrm{FaDu}$ cells.

In summary, our results not only identified the synergistic erlotinib-sensitizing drug pair of vorinostat and copanlisib, but also revealed the key mechanism of tridrug cooperation (Figure 6), thus providing a potential therapeutic drug combination for further clinical use.

\section{Acknowledgments}

Funding: National Natural Science Foundation of China (No. 82072997).

\section{Footnote}

Reporting Checklist: The authors have completed the MDAR reporting checklist. Available at https://dx.doi. org/10.21037/atm-21-4335

Data Sharing Statement: Available at https://dx.doi. org/10.21037/atm-21-4335

Conflicts of Interest: All authors have completed the ICMJE uniform disclosure form (available at https://dx.doi. org/10.21037/atm-21-4335). The authors have no conflicts of interest to declare.

Ethical Statement: The authors are accountable for all aspects of the work in ensuring that questions related to the accuracy or integrity of any part of the work are appropriately investigated and resolved.

Open Access Statement: This is an Open Access article 


\section{Page 12 of 13}

distributed in accordance with the Creative Commons Attribution-NonCommercial-NoDerivs 4.0 International License (CC BY-NC-ND 4.0), which permits the noncommercial replication and distribution of the article with the strict proviso that no changes or edits are made and the original work is properly cited (including links to both the formal publication through the relevant DOI and the license). See: https://creativecommons.org/licenses/by-nc-nd/4.0/.

\section{References}

1. Temam S, Kawaguchi H, El-Naggar AK, et al. Epidermal growth factor receptor copy number alterations correlate with poor clinical outcome in patients with head and neck squamous cancer. J Clin Oncol 2007;25:2164-70.

2. Rebucci M, Peixoto P, Dewitte A, et al. Mechanisms underlying resistance to cetuximab in the HNSCC cell line: role of AKT inhibition in bypassing this resistance. Int J Oncol 2011;38:189-200.

3. Cooper JB, Cohen EE. Mechanisms of resistance to EGFR inhibitors in head and neck cancer. Head Neck 2009;31:1086-94.

4. Soulieres D, Senzer NN, Vokes EE, et al. Multicenter phase II study of erlotinib, an oral epidermal growth factor receptor tyrosine kinase inhibitor, in patients with recurrent or metastatic squamous cell cancer of the head and neck. J Clin Oncol 2004;22:77-85.

5. Yun CH, Mengwasser KE, Toms AV, et al. The T790M mutation in EGFR kinase causes drug resistance by increasing the affinity for ATP. Proc Natl Acad Sci U S A 2008;105:2070-5.

6. Tafe LJ. Molecular mechanisms of therapy resistance in solid tumors: chasing "moving" targets. Virchows Arch 2017;471:155-64.

7. Kleszcz R, Skalski M, Krajka-Kuźniak V, et al. The inhibitors of KDM4 and KDM6 histone lysine demethylases enhance the anti-growth effects of erlotinib and HS-173 in head and neck cancer cells. Eur J Pharm Sci. 2021 Aug 8;166:105961.

8. Kurtz JE, Ray-Coquard I. PI3 kinase inhibitors in the clinic: an update. Anticancer Res 2012;32:2463-70.

9. Suda T, Hama T, Kondo S, et al. Copy number amplification of the PIK3CA gene is associated with poor prognosis in non-lymph node metastatic head and neck squamous cell carcinoma. BMC Cancer 2012;12:416.

10. Dreyling M, Santoro A, Mollica L, et al. Phosphatidylinositol 3-Kinase Inhibition by Copanlisib in Relapsed or Refractory Indolent Lymphoma. J Clin Oncol
Chen et al. Phospho-EGFRTyr992 attenuates resistance to erlotinib

2017;35:3898-905.

11. Pons-Tostivint E, Thibault B, Guillermet-Guibert J. Targeting PI3K Signaling in Combination Cancer Therapy. Trends Cancer 2017;3:454-69.

12. Giudice FS, Pinto DS Jr, Nör JE, et al. Inhibition of histone deacetylase impacts cancer stem cells and induces epithelial-mesenchyme transition of head and neck cancer. PLoS One 2013;8:e58672.

13. Chou CW, Wu MS, Huang WC, et al. HDAC inhibition decreases the expression of EGFR in colorectal cancer cells. PLoS One 2011;6:e18087.

14. Marks PA, Breslow R. Dimethyl sulfoxide to vorinostat: development of this histone deacetylase inhibitor as an anticancer drug. Nat Biotechnol 2007;25:84-90.

15. Wagner JM, Hackanson B, Lübbert $M$, et al. Histone deacetylase (HDAC) inhibitors in recent clinical trials for cancer therapy. Clin Epigenetics 2010;1:117-36.

16. Diaz N, Minton S, Cox C, et al. Activation of stat 3 in primary tumors from high-risk breast cancer patients is associated with elevated levels of activated SRC and survivin expression. Clin Cancer Res 2006;12:20-8.

17. Garcia R, Bowman TL, Niu G, et al. Constitutive activation of Stat3 by the Src and JAK tyrosine kinases participates in growth regulation of human breast carcinoma cells. Oncogene 2001;20:2499-513.

18. Pao W, Miller VA, Politi KA, et al. Acquired resistance of lung adenocarcinomas to gefitinib or erlotinib is associated with a second mutation in the EGFR kinase domain. PLoS Med 2005;2:e73.

19. Sette G, Salvati V, Mottolese M, et al. Tyr1068phosphorylated epidermal growth factor receptor (EGFR) predicts cancer stem cell targeting by erlotinib in preclinical models of wild-type EGFR lung cancer. Cell Death Dis 2015;6:e1850.

20. Zhang X, Liu G, Kang Y, et al. N-cadherin expression is associated with acquisition of EMT phenotype and with enhanced invasion in erlotinib-resistant lung cancer cell lines. PLoS One 2013;8:e57692.

21. Stabile LP, He G, Lui VW, et al. c-Src activation mediates erlotinib resistance in head and neck cancer by stimulating c-Met. Clin Cancer Res 2013;19:380-92.

22. Ma C, Wei S, Song Y. T790M and acquired resistance of EGFR TKI: a literature review of clinical reports. J Thorac Dis 2011;3:10-8.

23. Witta SE, Gemmill RM, Hirsch FR, et al. Restoring E-cadherin expression increases sensitivity to epidermal growth factor receptor inhibitors in lung cancer cell lines. Cancer Res 2006;66:944-50. 
24. Ocana A, Vera-Badillo F, Al-Mubarak M, et al. Activation of the PI3K/mTOR/AKT pathway and survival in solid tumors: systematic review and meta-analysis. PLoS One 2014;9:e95219.

25. Gao SP, Mark KG, Leslie K, et al. Mutations in the EGFR kinase domain mediate STAT3 activation via IL-6 production in human lung adenocarcinomas. J Clin Invest
2007;117:3846-56.

26. Lee HJ, Zhuang G, Cao Y, et al. Drug resistance via feedback activation of Stat 3 in oncogene-addicted cancer cells. Cancer Cell 2014;26:207-21.

(English Language Editor: A. Kassem)

Cite this article as: Chen X, Gao W, Yin G, Guo W, Huang J, Huang Z, Zhang Y. Phospho-EGFRTyr992 is synergistically repressed by co-inhibition of histone deacetylase (HDAC) and phosphatidylinositol 3-kinase (PI3K), which attenuates resistance to erlotinib in head and neck cancer cells. Ann Transl Med 2021;9(18):1455. doi: 10.21037/atm-21-4335 